\title{
Rosiglitazone attenuates cell apoptosis through antioxidative and anti-apoptotic pathways in the hippocampi of spontaneously hypertensive rats
}

\author{
YALI LI ${ }^{1}$, GUANGHU YU ${ }^{1}$, LIJUAN LIU ${ }^{1}$, JUN LONG ${ }^{1}$, SHUJIE SU ${ }^{1}$, \\ TING ZHAO $^{1}$, WENJIN LIU ${ }^{1}$, SHUNJI SHEN ${ }^{1}$ and XIAOLIN NIU ${ }^{2}$ \\ ${ }^{1}$ Department of Rehabilitation, Weihai Municipal Hospital, Weihai, Shandong 264200; ${ }^{2}$ Department of Cardiology, \\ The Second Affiliated Hospital, School of Medicine, Xi'an Jiaotong University, Xi'an, Shanxi 710004, P.R. China
}

Received February 11, 2018; Accepted September 27, 2018

DOI: 10.3892/ijmm.2018.3991

\begin{abstract}
Oxidative stress serves an important role in hypertensive brain damage. Peroxisome proliferator-activated receptor $\gamma$ (PPAR- $\gamma$ ) agonists possess antioxidative and anti-apoptotic effects. The present study verified the possibility that rosiglitazone serves a neuroprotective role by alleviating oxidative stress and cell apoptosis in the hippocampi of spontaneously hypertensive rats (SHRs). SHRs and age-matched Wistar-Kyoto (WKY; both 56 weeks old) rats received gavage administration of vehicle or rosiglitazone ( $5 \mathrm{mg} / \mathrm{kg} /$ day) for eight weeks. Systolic blood pressure (SBP) was measured by the indirect tail-cuff method. The expression ratio of activated astrocytes was analyzed by glial fibrillary acidic protein immunohistochemistry. PPAR- $\gamma$, inducible nitric oxide synthase (iNOS), gp47 $7^{\text {phox }}$, B-cell lymphoma 2 (Bcl-2), Bcl-2-associated X protein (Bax) and caspase-3 expression were investigated by quantitative polymerase chain reaction and western blot analysis. The number of apoptotic cells in the hippocampus of four groups was detected using the terminal deoxynucleotidyl transferase-mediated dUTP end-labeling (TUNEL) method. Compared with the WKY group, the SHR group exhibited decreased Bcl-2 and PPAR- $\gamma$ expression, increased SBP, increased ratio of activated astrocytes and TUNEL-positive cells, increased expression of iNOS, gp47 $7^{\text {phox }}$, caspase-3 and Bax. Rosiglitazone administration increased Bcl- 2 and PPAR- $\gamma$ expression, decreased the ratio of activated astrocytes and TUNEL-positive cells, decreased iNOS, gp $47^{\text {phox }}$, caspase-3 and Bax expression in the hippocampi of SHRs. However, rosiglitazone did not
\end{abstract}

Correspondence to: Dr Yali Li or Professor Shunji Shen, Department of Rehabilitation, Weihai Municipal Hospital, 248 Tong Yi Road, Weihai, Shandong 264200, P.R. China

E-mail: xjtuyali@stu.xjtu.edu.cn

E-mail: 724820063@qq.com

Key words: rosiglitazone, peroxisome proliferator-activated receptor $\gamma$, hypertension, oxidative stress, apoptosis significantly decreased SBP in the SHR group. Therefore, rosiglitazone exerts neuroprotective effect through antioxidative and anti-apoptotic pathways, which was independent of blood pressure control.

\section{Introduction}

Hypertension may induce brain damage primarily in the hippocampus and cortex area (1), which clinically manifest as cognitive decline. One of the main events that contribute toward this damage is the formation of reactive oxidative species (ROS) and reactive nitrogen species (RNS), and subsequent redox signaling disruption in mitochondria $(2,3)$, thereby resulting in enhanced oxidative stress. In fact, an enhancement of oxidative stress may decrease the level of neuronal activity and cause the influx of calcium ions into neurons, thereby activating the proapoptotic mechanisms (4), eventually resulting in neuronal apoptosis. However, there are no pharmacological approaches to limit or prevent this.

Peroxisome proliferator-activated receptor $\gamma$ (PPAR- $\gamma$ ) is a nuclear receptor family member, which regulates carbohydrate and lipid metabolism, and exerts antioxidative and anti-apoptotic actions in the central and peripheral nervous systems (5). A previous study indicated that PPAR- $\gamma$ ligands, including rosiglitazone and piglitazone, possess beneficial effects in cardiovascular disease, particularly in hypertension (6). Its well-characterized protective effects on hypertension (7) and target organs, including the brain (8), kidneys (9), heart (7) and vessels (10) prompted the use of PPAR- $\gamma$ agonists on hypertension-related organ damage. However, few studies have assessed the neuroprotective effect of rosiglitazone on hypertension-related cognitive decline in aged SHRs.

The results of our previous studies demonstrated that hypertension enhanced ox idative stress injury and exacerbated brain damage in the hippocampi of SHRs. Downregulated PPAR- $\gamma$ expression may be involved in this process (11). Furthermore, the present study aimed to examine whether the PPAR- $\gamma$ agonist, rosiglitazone, may exert neuroprotective effects in aged SHRs and investigate its underlying mechanisms of this. 


\section{Materials and methods}

Animals. A total of sixteen 56-week-old male SHRs and sixteen 56-week-old male Wistar-Kyoto (WKY) rats weighing $400 \mathrm{~g}$ (Shanghai Slac Laboratory Animal Center) were randomly assigned to control groups and rosiglitazone groups ( $\mathrm{n}=8$ per group). The rats received gavage administration of vehicle ( $0.9 \%$ saline) or rosiglitazone $(5 \mathrm{mg} / \mathrm{kg} / \mathrm{day})$ for eight weeks. All rats were housed in a room at $23^{\circ} \mathrm{C}, 60 \%$ humidity under a $12 \mathrm{~h} \mathrm{light/dark}$ cycle with free access to food and water. All the procedures were performed according to guidelines established by the Institutional Animal Care and Use Committee and the National Institutes of Health. The experimental protocols were approved by the Ethics Committee of Xi'an Jiaotong University College of Medicine (Xi'an, China) and followed the guidelines of the Declaration of Helsinki.

Blood pressure measurements. The indirect tail-cuff method (BP-2000; Visitech Systems, Inc., Apex, NC, USA) was used to measure systolic blood pressure (SBP). In brief, rats that had not been anesthetized were placed in a holding device mounted on a thermostatically-controlled warming plate to make the pulsations of the tail artery detectable. Tail cuffs were fixed on animals. SBP was measured at the end of the treatment period. Blood pressure was accurately recorded for each rat and averaged from $\geq 3$ consecutive readings.

Brain tissue preparation. Rats were anesthetized with $10 \%$ chloral hydrate $(400 \mathrm{mg} / \mathrm{kg})$ through intraperitoneal injection. No rats exhibited signs of peritonitis. For terminal deoxynucleotidyl transferase-mediated dUTP end-labeling (TUNEL) and immunohistochemistry assays, anesthetized rats $(n=4)$ were perfused transcardially with $0.9 \%$ saline (pH 7.4), followed by $4 \%$ paraformaldehyde (PFA). Next, the brains were post-fixed in 4\% PFA overnight. Targeted brain pieces were dehydrated, embedded in paraffin and cut into $10-\mu \mathrm{m}$-thick sections. Three sections containing the cortex and hippocampus were selected from each rat. For western blot analysis, anesthetized rats $(\mathrm{n}=4)$ were perfused transcardially with $0.9 \%$ saline ( $\mathrm{pH} 7.4$ ), prior to brains being removed and stored in sample protectors (Takara Biotechnology Co., Ltd., Dalian, China).

Reverse transcription-quantitative polymerase chain reaction $(R T-q P C R)$. Total RNA was extracted from the hippocampus using TRIzol reagent (Invitrogen; Thermo Fisher Scientific, Inc., Waltham, MA, USA) and the RNA samples were reverse transcribed to cDNA using the PrimeScript RT Master mix kit (Takara Biotechnology Co., Ltd.). All the procedures were performed according to the manufacturer's instructions. RT-qPCR was performed using the SYBR ExScript RT-PCR kit (Takara Biotechnology Co., Ltd.). The primer sequences for rat PPAR- $\gamma$ (forward, 5'-GGAGCCTAAGTTTGAGTT TGCTGTG-3' and reverse, 5'-TGCAGCAGGTTGTCTTGG ATG-3'); caspase-3 (forward, 5'-GAGACAGACAGTGGA ACTGACGATG-3' and reverse, 5'-GGCGCAAAGTGACTG GATGA-3'); B-cell lymphoma 2 (Bcl-2; forward, 5'-GAC TGAGTACCTGAACCGGCATC-3' and reverse, 5'-CTGAGC AGCGTCTTCAGAGACA-3'); Bcl-2-associated X protein
(Bax; forward, 5'-GCGTCCACCAAGAAGCTGA-3' and reverse, 5'-ACCACCCTGGTCTTGGATCC-3'); and $\beta$-actin (forward, 5'-GGAGATTACTGCCCTGGCTCCTA-3' and reverse, 5'-GACTCATCGTACTCCTGCTTGCTG-3') were designed and synthesized by Takara Biotechnology Co., Ltd. Amplification was performed at $95^{\circ} \mathrm{C}$ for $30 \mathrm{sec}$, followed by 40 cycles of $95^{\circ} \mathrm{C}$ for $3 \mathrm{sec}$ and $60^{\circ} \mathrm{C}$ for $30 \mathrm{sec}$. Cycle threshold values were obtained from the Bio-Rad iQ5 2.0 Standard Edition optical System software (Bio-Rad Laboratories, Inc., Hercules, CA, USA). Relative quantification was performed using the comparative method $\left(2^{-\Delta \Delta C q}\right)(12)$, and data are presented as the mean \pm standard deviation of three separate experiments performed in triplicate.

Immunohistochemistry. Sections were deparaffinized with serial xylene washes and rehydrated with serial concentrations of ethanol at room temperature (Xylene I, Xylene II, $100 \%$ alcohol I and 100\% alcohol II, 10 min each. Then, 95\% ethanol, $90 \%$ ethanol, $80 \%$ ethanol, $70 \%$ ethanol for $10 \mathrm{~min}$ each and distilled water). Then the sections were treated with $3 \%$ hydrogen peroxide for $20 \mathrm{~min}$, prior to incubation with $0.3 \%$ Triton X-100, followed by incubation with $10 \%$ goat serum for $1 \mathrm{~h}$. The slides were then incubated overnight at $4{ }^{\circ} \mathrm{C}$ with the rabbit polyclonal anti-glial fibrillary acidic protein (GFAP) antibody (1:4,000; cat. no. ab7260; Abcam, Cambridge, UK). Following three washes with PBS, the sections were incubated with a biotinylated anti-rabbit immunoglobulin $\mathrm{G}$ secondary antibody $(1: 1,000$; cat. no. ab150077; Abcam). 3,3'-diaminobenzidine was used to detect the immune reaction. For negative controls, the primary antibody was replaced with PBS. The slides were photographed under a light microscope (magnification, $\mathrm{x} 400$; Olympus Corporation, Tokyo, Japan). Digited images of tissue sections containing hippocampus were displayed on the video screen under identical lighting conditions. Labeled astrocytes were investigated in the left and right hippocampus from five sections per rat.

Western blot analysis. Radioimmunoprecipitation assay lysis buffer was used to extract total proteins from brain samples, followed by the measurement of protein concentrations using a bicinchoninic acid kit (Beyotime Institute of Biotechnology, Haimen, China). Equal amounts $(20 \mu \mathrm{g})$ of protein were separated on $12 \%$ SDS polyacrylamide gels, transferred to polyvinylidene difluoride membranes, and blocked in $10 \%$ skimmed milk for $2 \mathrm{~h}$ at $37^{\circ} \mathrm{C}$. Membranes were incubated with rabbit polyclonal antibodies against Bax, Bcl-2, gp47phox and caspase-3 (1:800; cat. nos. BS2538, BS6421, BS3261, P42574, respectively; Bioworld Technology, Inc., St. Louis Park, MN, USA), a rabbit polyclonal antibody against PPAR- $\gamma$ (1:400; cat. no. ab209350; Abcam) and a mouse monoclonal antibody against iNOS (1:500; cat. no. sc-7271; Santa Cruz Biotechnology, Inc., Dallas, TX, USA) overnight at $4^{\circ} \mathrm{C}$. Following three washes, the membranes were incubated with horseradish peroxidase (HRP)-conjugated goat anti-rabbit or goat anti-mouse antibody (1:10,000; cat. no. BS13278 and BS12478, respectively, Bioworld Technology, Inc.) for $2 \mathrm{~h}$ at $37^{\circ} \mathrm{C}$. Protein bands were detected using chemiluminescent HRP substrate (SuperSignal West Pico; Thermo Fisher Scientific, Inc.) for $5 \mathrm{~min}$ in a dark room and exposed to X-ray 

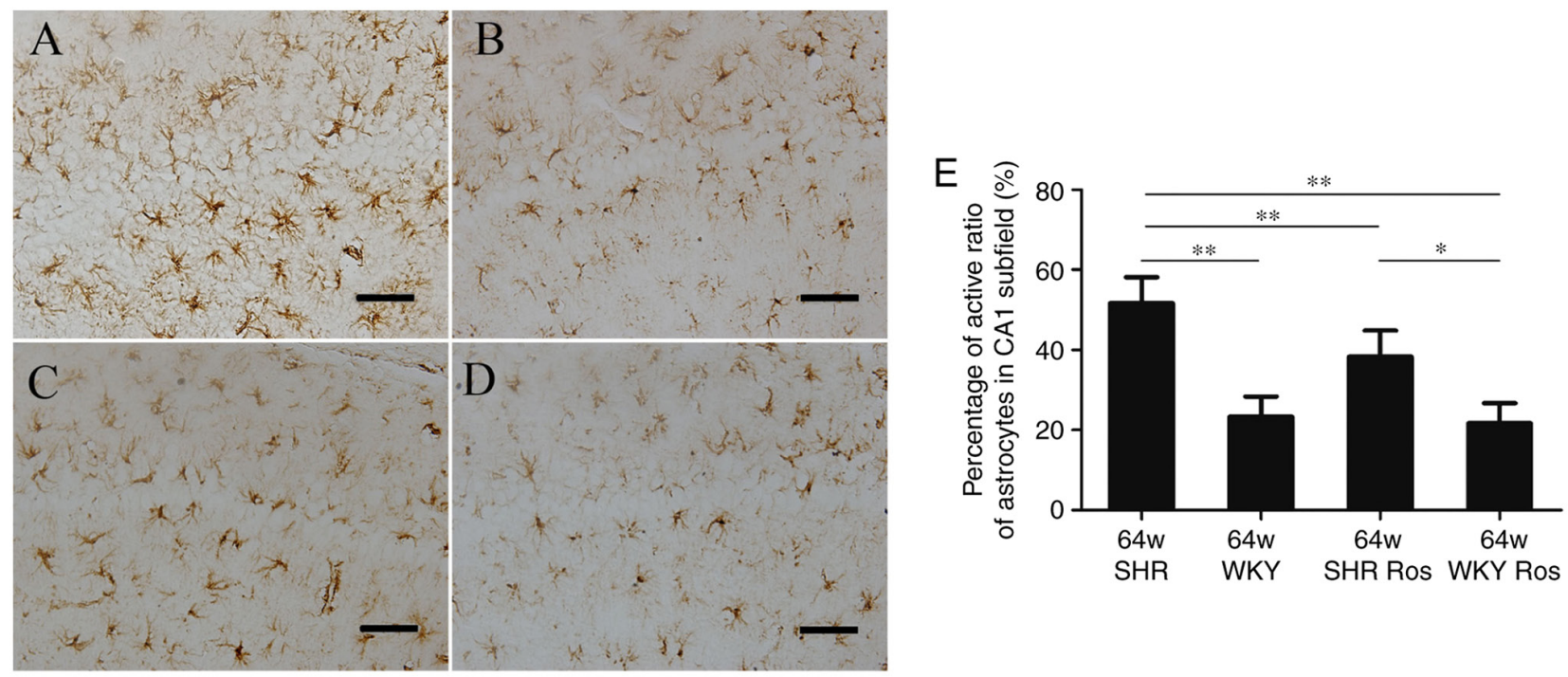

Figure 1. Sections of rat hippocampus processed for glial fibrillary acidic protein expression. Scale bar $=50 \mu \mathrm{m}$. The ratio of activated astrocytes was increased significantly in (A) the SHR group, compared with (B) the WKY group. Rosiglitazone decreased the activated ratio of astrocytes markedly in (C) the SHR group, but not in (D) the WKY group. (E) The parameters in the four groups. Columns and error bars represent the mean \pm standard error of the mean. "P<0.05, ${ }^{* *} \mathrm{P}<0.01$. SHR, spontaneously hypertensive rats; WKY, Wistar-Kyoto; ROS, rosiglitazone.

film (Fujifilm Corporation, Tokyo, Japan). The signal intensity of primary antibody binding was analyzed using Quantity One software 4.6.2 (Bio-Rad Laboratories, Inc.) and normalized to $\beta$-actin.

TUNEL assay. A cell death detection kit was used to perform the TUNEL assay (Promega Corporation, Madison, WI, USA) according to the manufacturer's protocols. In brief, the sections were dewaxed and rehydrated as described above. Then sections were fixed with $4 \%$ paraformaldehyde prepared in $0.1 \mathrm{M}$ PBS for $15 \mathrm{~min}$ at room temperature and washed in PBS for three times. The sections were then processed with $20 \mu \mathrm{g} / \mathrm{ml}$ fresh proteinase-K (diluted 1:500 in PBS) for $20 \mathrm{~min}$ at room temperature, followed by three washes in PBS. Next, sections were treated with equilibration buffer for $10 \mathrm{~min}$ at room temperature, followed by incubation with $50 \mu \mathrm{l}$ rTdT incubation buffer for $1 \mathrm{~h}$ at $37^{\circ} \mathrm{C}$ in the dark. Then slides were immersed in $2 \mathrm{X}$ SSC buffer for $15 \mathrm{~min}$ to terminate the reaction, followed by three washes with PBS. Sections were then counterstained with 4',6'-diamino-2-phenylindole (DAPI; dilution, 1:1,000; Sigma-Aldrich; Merck KGaA, Darmstadt, Germany). Fluorescence microscopy was performed using an Olympus BX51 microscope equipped with a mercury lamp power supply (magnification, $x 400$ ). Cells with bright green nuclei were identified as TUNEL-positive cells. TUNEL-positive cells were normalized to DAPI-stained cells. Immunoreactive cells from 9 randomly selected fields (3 samples per group, 3 fields per sample) were counted using a $\times 20$ objective lens by an observer blinded to the treatment groups.

Statistical analysis. Statistical analysis was performed using SPSS 16.0 software (SPSS, Inc., Chicago, IL, USA). The quantitative data are presented as the mean \pm standard deviation and analyzed using SPSS 16.0 software. Differences were detected by two-way analysis of variance (ANOVA), and then one-way ANOVA, followed by the least significant difference test was used to identify significant differences between the groups. Levene's test was used to test the homogeneity of variance and the distribution of normality. $\mathrm{P}<0.05$ was considered to indicate a statistically significant difference.

\section{Results}

Rosiglitazone does not significantly reduce $S B P$ in SHRs. The SBP in the 64-week-old SHR group averaged $193.8 \pm 11.2 \mathrm{mmHg}$, which was significantly different to WKY group $(110.8 \pm 9.7 \mathrm{mmHg} ; \mathrm{P}<0.01)$. The mean SBP levels were $106.5 \pm 4.3$ and $187.5 \pm 12.6 \mathrm{mmHg}$ in the rosiglitazone-treated WKY and SHR groups, respectively. However, rosiglitazone treatment did not have a significant effect on SBP $(\mathrm{P}>0.05)$. Therefore, rosiglitazone at the dose used in the present study did not decrease the SBP of the SHR group throughout the treatment period.

Rosiglitazone decreases the activated ratio of astrocytes in SHRs. As demonstrated in Fig. 1, the WKY group exhibited thin, shallow, dark-brown astrocytes with slender branches (Fig. 1B), while the SHR group developed more activated astrocytes with thick branches and large cell bodies (Fig. 1A). It was revealed that the two groups exhibited a similar number of astrocytes. However, the activated ratio of GFAP-positive astrocytes was increased in the CA1 subfield of the SHR group, compared with the WKY group (51.0 and 22.5\%, respectively; $\mathrm{P}<0.01)$. The parameter was decreased by rosiglitazone in the SHR group (37.8\%; P<0.01; Fig. 1E), but not in the WKY group (21.0\%; P>0.05; Fig. 1E).

Quantitative analysis of TUNEL-positive cells. As demonstrated in Fig. 2, neurons in the SHR group exhibited microanatomical characteristics of damaged cells, manifested with irregular cellular and nuclear profiles. A total of $46.1 \%$ TUNEL-positive nuclei were observed in the SHR group (Fig. 2A). Rosiglitazone 

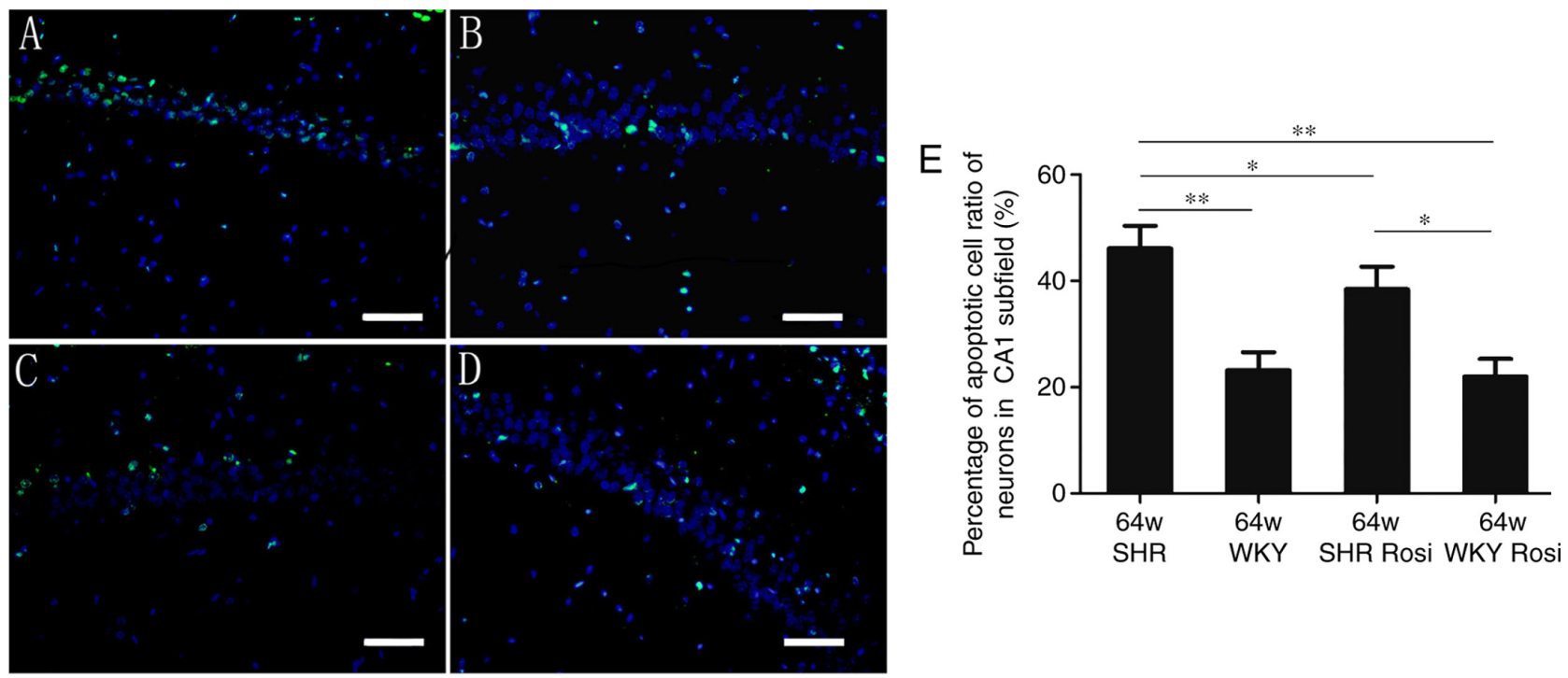

Figure 2. Sections of rat hippocampus processed for TUNEL staining. Scale bar=50 $\mu \mathrm{m}$. (A) The SHR group exhibited $46.1 \%$ TUNEL-positive nuclei, which was significantly different to (B) the WKY group (23.2\%). Rosiglitazone decreased the number of apoptotic cells in (C) the SHR group (38.4\%), but not in (D) the WKY group (22.0\%). (E) The parameters in the four groups. Columns and error bars represent the mean \pm standard error of the mean. ${ }^{*} \mathrm{P}<0.05,{ }^{* *} \mathrm{P}<0.01$. TUNEL, terminal deoxynucleotidyl transferase-mediated dUTP end-labeling; SHR, spontaneously hypertensive rats; WKY, Wistar-Kyoto; ROS, rosiglitazone; PPAR- $\gamma$, peroxisome proliferator-activated receptor $\gamma$.

decreased this number to $38.4 \%$ (Fig. 2C), which revealed significant differences with the SHR group $(\mathrm{P}<0.05)$. In the hippocampi of the WKY group, $\sim 23.2 \%$ of the nuclei were TUNEL-positive (Fig. 2B). The parameter was decreased to $22.0 \%$ by rosiglitazone (Fig. 2D). The differences between the two groups were not statistically significant $(\mathrm{P}>0.05$; Fig. $2 \mathrm{E})$.

Rosiglitazone increases PPAR- $\gamma$ expression in the hippocampi of SHRs. As demonstrated in Fig. 3, hypertension and rosiglitazone treatment have significant effects on PPAR- $\gamma$ expression $(\mathrm{P}<0.01)$. Compared with the WKY group, the SHR group exhibited significantly decreased mRNA and protein expression of PPAR $-\gamma(\mathrm{P}<0.01)$. Rosiglitazone significantly increased PPAR- $\gamma$ mRNA and protein expression in the SHR group $(\mathrm{P}<0.01)$, but not in the WKY group $(\mathrm{P}>0.05)$.

Rosiglitazone attenuates oxidative stress injury in SHRs. As demonstrated in Fig. 4, hypertension and rosiglitazone treatment altered the protein expression of iNOS and gp $47^{\text {phox }}$ $(\mathrm{P}<0.01)$. The SHR group exhibited significantly upregulated protein expression of iNOS and gp4 $7^{\text {phox }}$ compared with the WKY group $(\mathrm{P}<0.01)$. Rosiglitazone significantly decreased iNOS and gp47 phox expression in the SHR group $(\mathrm{P}<0.01)$, but not in the WKY group $(\mathrm{P}>0.05)$.

Rosiglitazone exerts anti-apoptotic effects in SHRs. As demonstrated in Fig. 5, compared with the WKY group, Bcl-2 mRNA expression was downregulated, while Bax and caspase-3 mRNA expression were upregulated in the SHR group $(\mathrm{P}<0.01)$. Rosiglitazone did not alter Bax and Bcl-2 mRNA expression $(\mathrm{P}>0.05)$, but significantly decreased caspase- 3 mRNA expression $(\mathrm{P}<0.01)$ in the SHR group.

The SHR group exhibited increased protein expression of Bax and caspase- 3 , and upregulated the ratio of $\mathrm{Bax} / \mathrm{Bcl}-2$ (Fig. 6). The indexes in the SHR group all significantly differed from the WKY group $(\mathrm{P}<0.01)$. By contrast, the
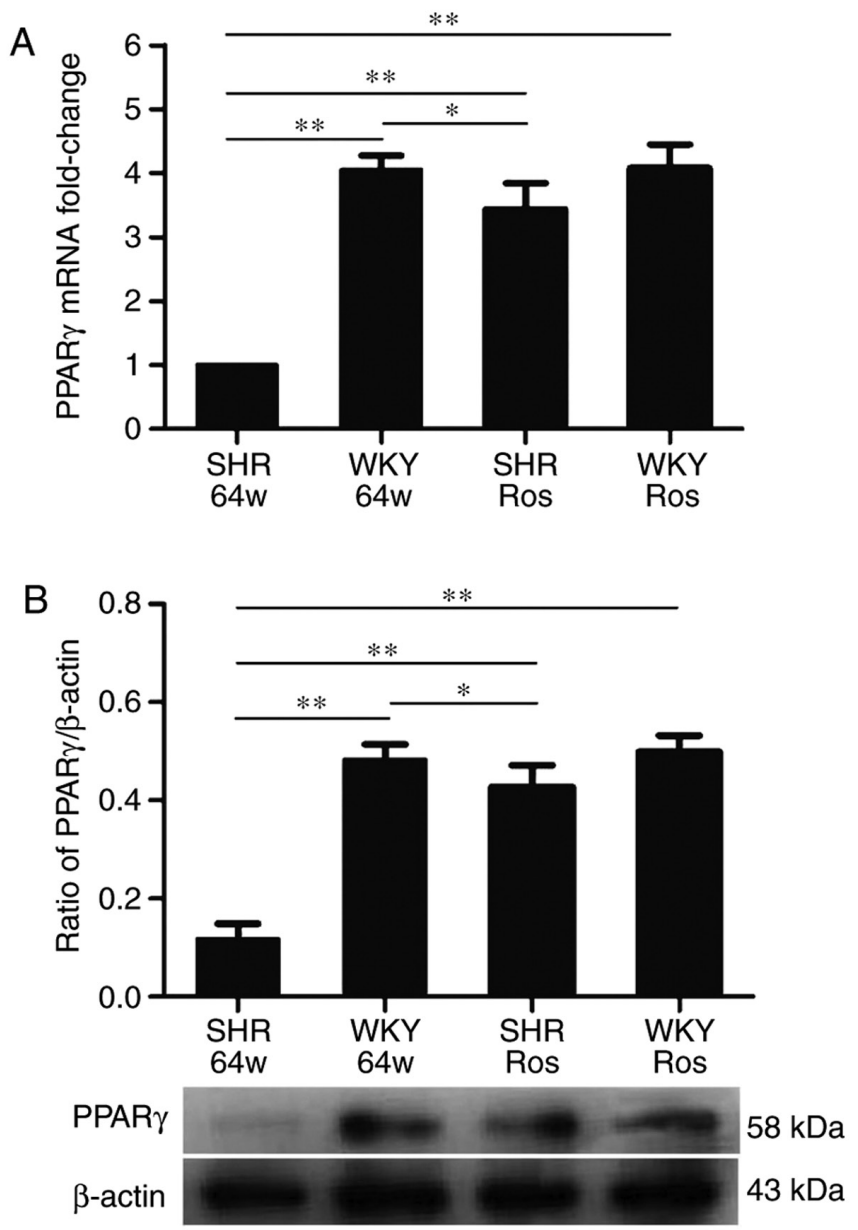

Figure 3. PPAR $-\gamma$ mRNA and protein expression in four groups. The SHR group exhibited decreased PPAR- $\gamma$ (A) mRNA and (B) protein expression. Rosiglitazone markedly upregulated PPAR- $\gamma$ (A) mRNA and (B) protein expression in the SHR group, but not in the WKY group. Columns and error bars represent the mean \pm standard error of the mean. ${ }^{*} \mathrm{P}<0.05,{ }^{* *} \mathrm{P}<0.01$. PPAR- $\gamma$, peroxisome proliferator-activated receptor $\gamma$; SHR, spontaneously hypertensive rats; WKY, Wistar-Kyoto; ROS, rosiglitazone. 

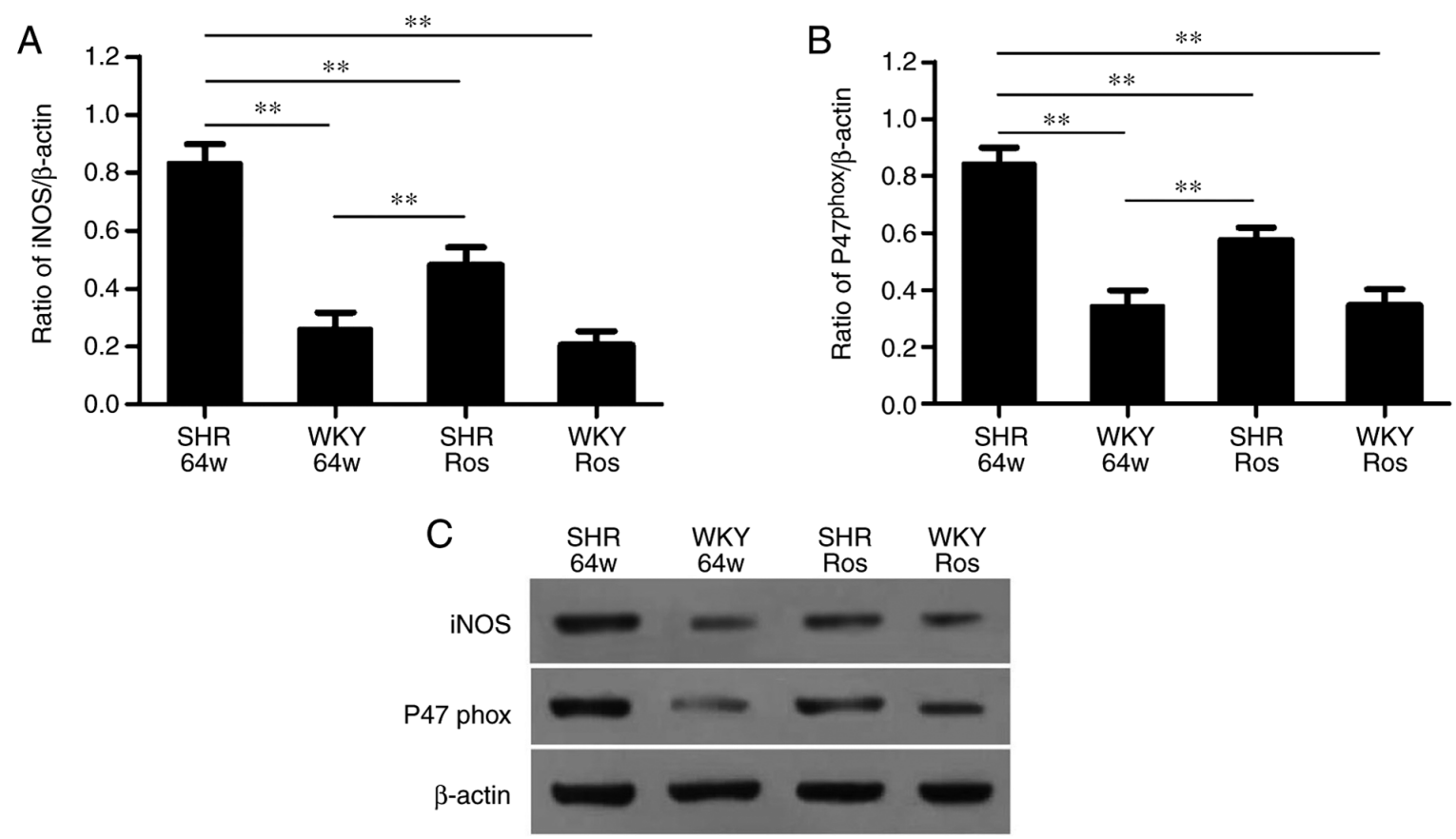

Figure 4. Protein expression of gp47 $7^{\text {phox }}$ and iNOS in four groups. The SHR group exhibited upregulated (A) iNOS and (B) gp47 $7^{\text {phox }}$ protein expression. Rosiglitazone decreased (A) iNOS and (B) gp47 phox expression in the SHR group, but not in the WKY group. (C) Representative western blotting image, displaying iNOS and gp47 phox protein expression. Columns and error bars represent the mean \pm standard error of the mean. ${ }^{* * *} \mathrm{P}<0.01$. iNOS, inducible nitric oxide synthase; SHR, spontaneously hypertensive rats; WKY, Wistar-Kyoto; ROS, rosiglitazone.

A

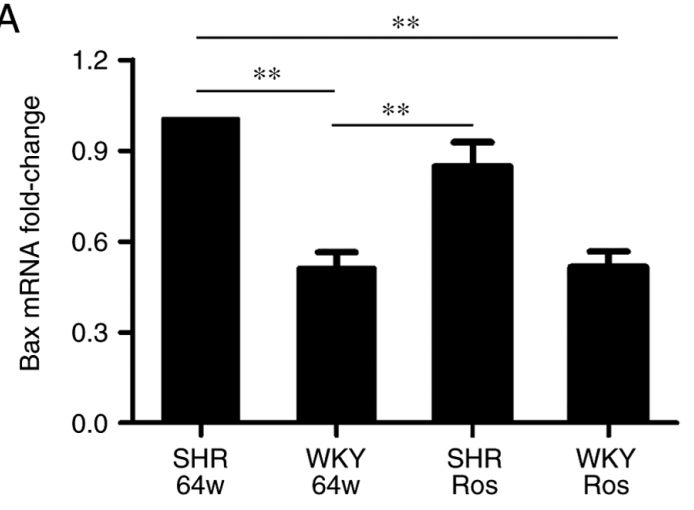

B

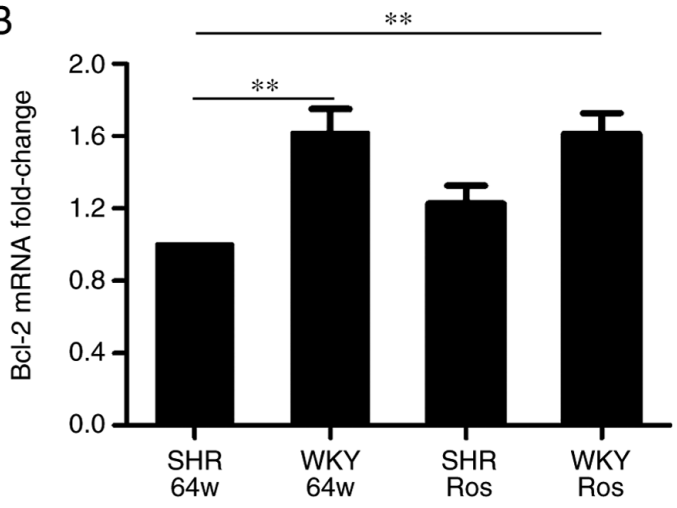

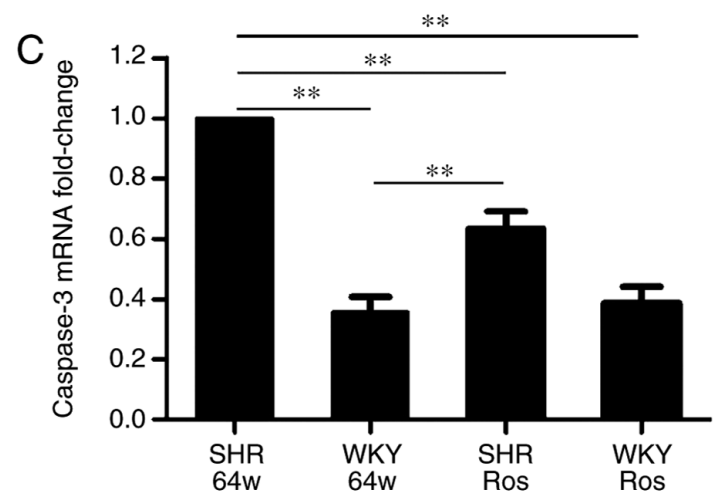

Figure 5. Expression levels of Bcl-2, Bax and caspase-3 mRNA in four groups. The SHR group exhibited (B) downregulated Bcl-2 mRNA, and (A) upregulated Bax and (C) caspase-3 mRNA expression. Rosiglitazone did not alter (A) Bax and (B) Bcl-2 mRNA expression, but significantly decreased (C) caspase-3 mRNA expression in the SHR group. Columns and error bars represent the mean \pm standard error of the mean. ${ }^{* *} \mathrm{P}<0.01$. Bcl-2, B-cell lymphoma 2; Bax, Bcl-2-associated X protein; SHR, spontaneously hypertensive rats; WKY, Wistar-Kyoto; ROS, rosiglitazone.

protein expression of Bcl-2 was decreased in the SHR group $(\mathrm{P}<0.01)$. Rosiglitazone significantly decreased Bax, caspase-3 protein expression $(\mathrm{P}<0.05$ and $\mathrm{P}<0.01$, respectively) and the $\mathrm{Bax} / \mathrm{Bcl}-2$ expression ratio $(\mathrm{P}<0.01)$. 

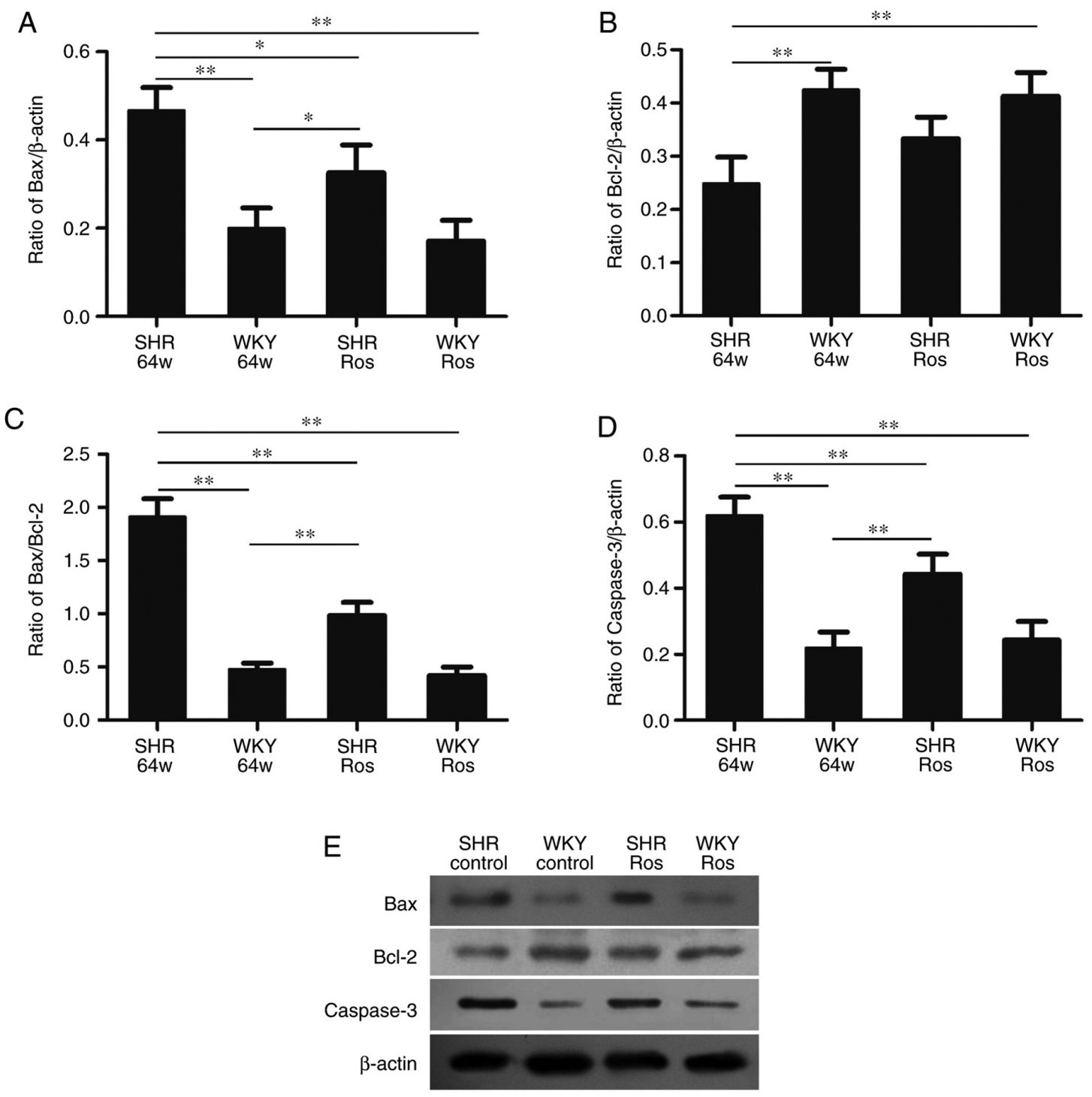

Figure 6. Protein expression of Bcl-2, Bax and caspase-3 in four groups. SHR group exhibited (B) downregulated Bcl-2 protein, and (A) upregulated Bax, (D) caspase-3 protein expression and (C) Bax/Bcl-2 expression ratio. Rosiglitazone decreased (A) Bax and (D) caspase-3 protein expression and (C) Bax/Bcl-2 expression ratio, but did not increase (B) Bcl-2 protein expression in the SHR or WKY groups. (E) Representative western blotting image, displaying Bax, Bcl-2 and caspase-3 protein expression. Columns and error bars represent the mean \pm standard error of the mean. ${ }^{*} \mathrm{P}<0.05,{ }^{* *} \mathrm{P}<0.01$. Bcl-2, B-cell lymphoma 2; Bax, Bcl-2-associated X protein; SHR, spontaneously hypertensive rats; WKY, Wistar-Kyoto; ROS, rosiglitazone.

\section{Discussion}

The results of our previous study demonstrated that SHRs exhibited an age-dependent increase in TUNEL-positive cells in the CA1 subfield of the hippocampus, which was accompanied with increased expression of oxidative stress markers and decreased mRNA and protein expression of PPAR- $\gamma$ (11). Therefore, we hypothesized that downregulated PPAR- $\gamma$ expression may be the underlying mechanism of hypertension-related brain damage. In order to verify this hypothesis, 64-week-old SHRs and age-matched WKY rats were used to study whether the PPAR- $\gamma$ agonist, rosiglitazone, may exert neuroprotective effects on aged SHRs and the underlying mechanism of this was investigated. To the best of our knowledge, rosiglitazone has been extensively studied in vessels (13), kidneys (13) and the heart (14) of SHRs. Few studies have investigated the effect of rosiglitazone on hypertensive brain damage in aged SHRs. The main results of the present study were that aged SHRs exhibited exacerbated oxidative stress injury in the hippocampi, and that the PPAR- $\gamma$ agonist, rosiglitazone, exerts neuroprotective effects through antioxidative and anti-apoptotic pathways in preventing SHRs from brain damage, which was independent of blood pressure control.

Oxidative stress results from an imbalance of production over degradation of ROS and is associated with hypertension (15). It is also one of the main pathophysiological mechanisms of hypertensive brain damage. It is well known that ROS derives mainly from NADPH oxidase enzymatic activity in brain tissues (16). Therefore, the present study evaluated NADPH oxidase subunit gp $47^{\text {phox }}$ expression in the SHR and WKY groups in order to analyze the impact of hypertension on the activation of NADPH oxidase in the hippocampus and its role in oxidative stress. Additionally, iNOS is another important marker of oxidative stress, is observed in neurons and is selectively distributed in different brain regions. Once induced, iNOS can generate toxic levels of nitric oxide, 
resulting in cell damage and death in the brain (17). The present study revealed that the expression of gp47phox and iNOS was significantly increased in the hippocampi of the SHR group. The increased protein expression levels of the two parameters provided additional support for increased ROS production and aggravated oxidative stress injury in the hippocampi of aged SHRs. It is well known that oxidative stress damages neurons. Excessive ROS may attack cellular components, including proteins, lipids and DNA, and may cause cell death via necrosis or apoptosis (18). We hypothesized that this state of oxidative stress may increase the susceptibility of neurons to apoptosis and represent a risk factor for neuronal death.

In line with the occurrence of aggravated oxidative stress changes in the hippocampi of SHRs, similar results were also observed following TUNEL staining, which was used to detect the rupture of cell nuclei DNA during the early apoptotic process of tissue cells. However, it was suggested that the specificity of the TUNEL technique for apoptosis may also identify necrotic nuclei (19). In the present study, the SHR group exhibited a significantly increased number of TUNEL-positive cells, compared with the WKY group. Although it is not possible to conclude whether or not cells of the CA1 subfield undergo apoptosis and/or necrosis based on the present TUNEL data, the observation of more TUNEL-positive nuclei in the CA1 subfield of the SHR group indicated the occurrence of neurodegenerative changes in the hippocampi of aged SHRs. It is well known that the nerve cells in the hippocampus have the ability to receive, process and store information (20). The observation of fewer cells or more TUNEL-positive cells in the aged SHRs suggested that brain function may undergo a chronic degenerative changes.

Astrocytes are activated in the condition of brain injury by increasing the number of their intermediate filaments and stain strongly for GFAP (21). Therefore, increased expression of immunoreactivity of GFAP is considered to be an indicator of brain injury. In the present study, the SHR group exhibited an increased percentage of activated astrocytes with thick branches and large cell bodies. The presence of more activated astrocytes in 64-week-old SHRs probably indicated that the hippocampus was impaired in the hypertensive state and the increase in the activated astrocytes maybe a compensatory protection method for prevent damage to brain tissue.

PPAR $-\gamma$ is a ligand-activated transcription factor, which serves beneficial roles in inhibiting oxidative stress in the central nervous system (22). Once activated by its agonist, PPAR- $\gamma$ can protect QZG cells against oxidative stress injury (23). Therefore, PPAR- $\gamma$ agonists may exert beneficial effects in oxidative stress-related brain injury. Our previous study demonstrated that SHRs exhibit an age-dependent reduction in PPAR- $\gamma$ expression in the hippocampus (11), which suggested that downregulated PPAR- $\gamma$ expression may underline hypertensive brain damage. In the present study, rosiglitazone administration upregulated PPAR $-\gamma$ mRNA and protein expression in aged SHRs, which was accompanied by markedly decreased expression of oxidative stress markers (iNOS and gp47 $7^{\text {phox }}$ ) and pro-apoptotic markers (Bax and caspase-3). Rosiglitazone also decreased the activated ratio of astrocytes and decreased the number of TUNEL-positive cells in the hippocampi of aged SHRs. These results indicated that the PPAR- $\gamma$ agonist, rosiglitazone, may protect the neuronal microenvironment and preserve nerve cells, thereby exerting neuroprotective effects through antioxidative and anti-apoptotic mechanisms.

It is noteworthy that in the present study, rosiglitazone did not significantly decrease SBP in the SHR group. Accumulating evidence has indicated that rosiglitazone possesses potent blood pressure-lowering effects in patients (24) and animal models with metabolic syndrome (25). The underlying mechanisms of this have not yet been fully elucidated, including inhibiting the proliferation of arterial smooth muscle cells (26), protecting endothelial-dependent vasodilation (27), increasing nitrous oxide production availability (28) and alleviating oxidative stress in the rostral ventrolateral medulla of the SHRs (29). A possible limitation of the present study is that the dose used was $5 \mathrm{mg} / \mathrm{kg} / \mathrm{day}$, which was different from previous studies, which used $20 \mathrm{mg} / \mathrm{kg} /$ day (29) or $50 \mathrm{mmol} / \mathrm{kg} /$ day (30). It was concluded that the effect of rosiglitazone is dose-dependent and that the dose in the present study was insufficient to result in a blood pressure-lowering effect.

In conclusion, the results of the present study demonstrated that aged SHRs exhibited increased apoptotic cells in the hippocampus, accompanied by increased levels of oxidative stress markers and pro-apoptotic markers, suggesting the important role of oxidative stress injury in hypertensive brain damage. The PPAR- $\gamma$ agonist, rosiglitazone, may protect neuronal microenvironment and preserve nerve cells in the CA1 subfield of hippocampi of SHRs through antioxidative and anti-apoptotic pathways, which are independent of blood pressure control. Another are that 5 requires further investigation is to assess whether other glial cell populations, besides astrocytes, participate in the mechanism of hypertensive brain damage.

\section{Acknowledgements}

The authors would like to thank Professor Yi Zhu for providing technical assistance.

\section{Funding}

The present study was supported by the National Natural Science Foundation of China (grant no. 81070219).

\section{Availability of data and materials}

The datasets generated and/or analyzed during the current study are not publicly available as the data form part of an ongoing study, but are available from the corresponding author on reasonable request.

\section{Authors' contributions}

YL and XN conceived the study. YL, TZ and WL performed the experiments and collected the data. SSh, LL, GY, JL and SSu analyzed and interpreted the data. YL drafted the paper. YL and SSh edited and revised the manuscript. YL reviewed the manuscript. All the authors read and approved the final manuscript.

\section{Ethics approval and consent to participate}

The methods of specimen collection were conducted in accordance with the guidelines of National Institutes of Health. 
The experimental protocols were approved by the Ethics Committee of Xi'an Jiaotong University College of Medicine (Xi'an, China) and were performed according to the guidelines of the Declaration of Helsinki.

\section{Patient consent for publication}

Not applicable.

\section{Competing interests}

The authors declare that they have no competing interests.

\section{References}

1. Selvetella G, Notte A, Maffei A, Calistri V, Scamardella V, Frati G, Trimarco B, Colonnese C and Lembo G: Left ventricular hypertrophy is associated with asymptomatic cerebral damage in hypertensive patients. Stroke 34: 1766-1770, 2003.

2. Turer AT and Hill JA: Pathogenesis of myocardial ischemia-reperfusion injury and rationale for therapy. Am J Cardiol 106: $360-368,2010$

3. Eltzschig HK and Eckle T: Ischemia and reperfusion-from mechanism to translation. Nat Med 17: 1391-1401, 2011.

4. Mohammadi MT and Dehghani GA: Acute hypertension induces brain injury and blood-brain barrier disruption through reduction of claudins mRNA expression in rat. Pathol Res Pract 210: 985-990, 2014.

5. Corona JC and Duchen MR: PPARgamma as a therapeutic target to rescue mitochondrial function in neurological disease. Free Radic Biol Med 100: 153-163, 2016.

6. Kvandova M, Majzunova $M$ and Dovinova I: The role of PPARgamma in cardiovascular diseases. Physiol Res 65: S343-S363, 2016.

7. Chaudhry A, Carthan KA, Kang BY, Calvert J, Sutliff RL and Michael Hart C: PPARgamma attenuates hypoxia-induced hypertrophic transcriptional pathways in the heart. Pulm Circ 7: 98-107, 2017

8. Lan LF, Zheng L, Yang X, Ji XT, Fan YH and Zeng JS: Peroxisome proliferator-activated receptor-gamma agonist pioglitazone ameliorates white matter lesion and cognitive impairment in hypertensive rats. CNS Neurosci Ther 21: 410-416, 2015.

9. Afzal S, Sattar MA, Johns EJ, Abdulla MH, Akhtar S, Hashmi F and Abdullah NA: Interaction between irbesartan, peroxisome proliferator-activated receptor (PPAR-gamma), and adiponectin in the regulation of blood pressure and renal function in spontaneously hypertensive rats. J Physiol Biochem 72: 593-604, 2016

10. Maccallini C, Mollica A and Amoroso R: The positive regulation of eNOS signaling by PPAR agonists in cardiovascular diseases. Am J Cardiovasc Drugs 17: 273-281, 2017.

11. Li Y, Liu J, Gao D, Wei J, Yuan H, Niu X and Zhang Q Age-related changes in hypertensive brain damage in the hippocampi of spontaneously hypertensive rats. Mol Med Rep 13 2552-2560, 2016.

12. Livak KJ and Schmittgen TD: Analysis of relative gene expression data using real-time quantitative PCR and the $2-\Delta \Delta \mathrm{Ct}$ method. Methods 25: 402-408, 2001.

13. Imig JD, Walsh KA, Hye Khan MA, Nagasawa T, CherianShaw M, Shaw SM and Hammock BD: Soluble epoxide hydrolase inhibition and peroxisome proliferator activated receptor gamma agonist improve vascular function and decrease renal injury in hypertensive obese rats. Exp Biol Med (Maywood) 237: $1402-1412,2012$.
14. Kadiiska MB, Bonini MG, Ruggiero C, Cleland E, Wicks S and Stadler K: Thiazolidinedione treatment decreases oxidative stress in spontaneously hypertensive heart failure rats through attenuation of inducible nitric oxide synthase-mediated lipid radical formation. Diabetes 61: 586-596, 2012.

15. Paravicini TM and Touyz RM: Redox signaling in hypertension. Cardiovasc Res 71: 247-258, 2006.

16. Patel M, Li QY, Chang LY, Crapo J and Liang LP: Activation of NADPH oxidase and extracellular superoxide production in seizure-induced hippocampal damage. J Neurochem 92: 123-131, 2005.

17. Jiang T, Gao L, Shi J,Lu J, Wang Y and Zhang Y: Angiotensin-(1-7) modulates renin-angiotensin system associated with reducing oxidative stress and attenuating neuronal apoptosis in the brain of hypertensive rats. Pharmacol Res 67: 84-93, 2013.

18. Ramalingam M and Kim SJ: Reactive oxygen/nitrogen species and their functional correlations in neurodegenerative diseases. J Neural Transm (Vienna) 119: 891-910, 2012.

19. Charriaut-Marlangue $C$ and Ben-Ari Y: A cautionary note on the use of the TUNEL stain to determine apoptosis. Neuroreport 7 : 61-64, 1995.

20. Napoleone P, Ferrante F, Ghirardi O, Ramacci MT and Amenta F: Age-dependent nerve cell loss in the brain of Sprague-Dawley rats: Effect of long term acetyl-L-carnitine treatment. Arch Gerontol Geriatr 10: 173-185, 1990.

21. Beach TG, Walker R and McGeer EG: Patterns of gliosis in Alzheimer's disease and aging cerebrum. Glia 2: 420-436, 1989.

22. Kvandová M, Majzúnová M and Dovinová I: The role of PPARgamma in cardiovascular diseases. Physiol Res 65: S343-S363, 2016.

23. Li WL, Liang X, Wang X, Zhang XD, Liu R, Zhang W, Chen HL, Qin XJ, Bai H and Hai CX: Protective effect of the peroxisome proliferator-activated receptor (PPAR)-gamma, ligand rosiglitazone on tert-butyl hydroperoxide-induced QZG cell injury. Exp Toxicol Pathol 63: 527-533, 2011.

24. Raji A, Seely EW, Bekins SA, Williams GH and Simonson DC: Rosiglitazone improves insulin sensitivity and lowers blood pressure in hypertensive patients. Diabetes Care 26: 172-178, 2003.

25. Dobrian AD, Schriver SD, Khraibi AA and Prewitt RL: Pioglitazone prevents hypertension and reduces oxidative stress in diet-induced obesity. Hypertension 43: 48-56, 2004.

26. de Dios ST, Bruemmer D, Dilley RJ, Ivey ME, Jennings GL, Law RE and Little PJ: Inhibitory activity of clinical thiazolidinedione peroxisome proliferator activating receptor-gamma ligands toward internal mammary artery, radial artery, and saphenous vein smooth muscle cell proliferation. Circulation 107: 2548-2550, 2003.

27. Wang TD, Chen WJ, Cheng WC, Lin JW, Chen MF and Lee YT: Relation of improvement in endothelium-dependent flow-mediated vasodilation after rosiglitazone to changes in asymmetric dimethylarginine, endothelin-1, and C-reactive protein in nondiabetic patients with the metabolic syndrome. Am J Cardiol 98: 1057-1062, 2006

28. Polikandriotis JA, Mazzella LJ, Rupnow HL and Hart CM: Peroxisome proliferator-activated receptor gamma ligands stimulate endothelial nitric oxide production through distinct peroxisome proliferator-activated receptor gamma-dependent mechanisms. Arterioscler Thromb Vasc Biol 25: 1810-1816, 2005.

29. Chan SH, Wu KL, Kung PS and Chan JY: Oral intake of rosiglitazone promotes a central antihypertensive effect via upregulation of peroxisome proliferator-activated receptor-gamma and alleviation of oxidative stress in rostral ventrolateral medulla of spontaneously hypertensive rats. Hypertension 55: 1444-1453, 2010.

30. Walker AB, Chattington PD, Buckingham RE and Williams G: The thiazolidinedione rosiglitazone (BRL-49653) lowers blood pressure and protects against impairment of endothelial function in Zucker fatty rats. Diabetes 48: 1448-1453, 1999. 